\title{
Thoracoscopic lobectomy is associated with lower morbidity compared with thoracotomy
}

\author{
Nestor R. Villamizar, MD, ${ }^{\text {a }}$ Marcus D. Darrabie, MD, ${ }^{a}$ William R. Burfeind, MD, ${ }^{\mathrm{b}}$ \\ Rebecca P. Petersen, MD, ${ }^{a}$ Mark W. Onaitis, MD, ${ }^{a}$ Eric Toloza, MD, ${ }^{a}$ David H. Harpole, MD, ${ }^{a}$ and \\ Thomas A. D'Amico, MD
}

\begin{abstract}
Objectives: Advantages of thoracoscopic lobectomy include less postoperative pain, shorter hospitalization, and improved delivery of adjuvant chemotherapy. The incidence of postoperative complications has not been thoroughly assessed. This study analyzes morbidity after lobectomy to compare the thoracoscopic approach and thoracotomy.
\end{abstract}

Methods: By using a prospective database, the outcomes of patients who underwent lobectomy from 1999-2009 were analyzed with respect to postoperative complications. Propensity-matched groups were analyzed based on preoperative variables and stage.

Results: Of the 1079 patients in the study, 697 underwent thoracoscopic lobectomy, and 382 underwent lobectomy by means of thoracotomy. In the overall analysis thoracoscopic lobectomy was associated with a lower incidence of atrial fibrillation $(P=.01)$, atelectasis $(P=.0001)$, prolonged air leak $(P=.0004)$, transfusion $(P=.0001)$, pneumonia $(P=.001)$, sepsis $(P=.008)$, renal failure $(P=.003)$, and death $(P=.003)$. In the propensity-matched analysis based on preoperative variables, when comparing 284 patients in each group, 196 $(69 \%)$ patients who underwent thoracoscopic lobectomy had no complications versus $144(51 \%)$ patients who underwent thoracotomy $(P=.0001)$. In addition, thoracoscopic lobectomy was associated with a lower incidence of atrial fibrillation ( $13 \%$ vs $21 \%, P=.01)$, less atelectasis $(5 \%$ vs $12 \%, P=.006)$, fewer prolonged air leaks $(13 \%$ vs $19 \%, P=.05)$, fewer transfusions $(4 \%$ vs $13 \%, P=.002)$, less pneumonia $(5 \%$ vs $10 \%$, $P=.05)$, less renal failure $(1.4 \%$ vs $5 \%, P=.02)$, shorter chest tube duration (median of 3 vs 4 days, $P<.0001$ ), and shorter length of hospital stay (median of 4 vs 5 days, $P<.0001$ ).

Conclusions: Thoracoscopic lobectomy is associated with a lower incidence of major complications, including atrial fibrillation, compared with lobectomy by means of thoracotomy. The underlying factors responsible for this advantage should be analyzed to improve the safety and outcomes of other thoracic procedures.

Earn CME credits at

http://cme.ctsnetjournals.org
Thoracoscopic lobectomy has been successfully performed worldwide for more than a decade, has emerged as a reasonable option for the management of early-stage non-small cell lung cancer (NSCLC), and is supported by evidencebased treatment guidelines. ${ }^{1-4}$ Advantages of thoracoscopic lobectomy compared with thoracotomy include less postop-

\footnotetext{
From the Department of Surgery, ${ }^{\mathrm{a}}$ Duke University Medical Center, Durham, NC; and Department of Thoracic Surgery, ${ }^{\text {b }}$ St Luke's Health Network, Bethlehem, Pa.

Read at the Thirty-fourth Annual Meeting of the Western Thoracic Surgical Association, Kona, Hawaii, June 25-28, 2008.

Received for publication June 24, 2008; revisions received April 7, 2009; accepted for publication April 24, 2009.

Address for reprints: Thomas A. D'Amico, MD, Department of Surgery, Duke University Medical Center Box 3496, Duke South, White Zone, Room 3589, Durham, NC 27710 (E-mail: damic001@mc.duke.edu).

J Thorac Cardiovasc Surg 2009;138:419-25

$0022-5223 / \$ 36.00$

Copyright (C) 2009 by The American Association for Thoracic Surgery

doi:10.1016/j.jtcvs.2009.04.026
}

erative pain, ${ }^{5,6}$ shorter hospitalization, ${ }^{2,3,7,8}$ and improved delivery of adjuvant chemotherapy to eligible patients. ${ }^{9,10}$

Despite these outcomes, the advantages of thoracoscopic lobectomy appear to be underestimated. From 1999-2006, only $20 \%$ of all lobectomies for NSCLC were performed thoracoscopically by the board-certified thoracic surgeons participating in the general thoracic surgery component of the Society of Thoracic Surgeons database. ${ }^{11}$ Recently, morbidity and mortality after thoracoscopic lobectomy have been assessed, demonstrating improved results for some but not all outcomes. ${ }^{12-14}$ In the present study postoperative morbidity and mortality after lobectomy are analyzed by using a large, prospectively managed database to compare outcomes after the thoracoscopic approach and thoracotomy by using a propensity-matched analysis.

\section{MATERIALS AND METHODS \\ Patient Selection}

After obtaining approval from the institutional review board and waivers of informed consent, a prospectively maintained database of all patients who underwent lobectomy between February 1999 and October 2008 was queried. Subsequently, a retrospective review of all prospectively collected data was performed for each individual case. The sources of information that were reviewed included the institutional Society of Thoracic Surgeons 


\section{Abbreviations and Acronyms \\ $\mathrm{AF} \quad=$ atrial fibrillation \\ MI = myocardial infarction \\ NSCLC $=$ non-small cell lung cancer}

database, the preoperative clinic note, preoperative studies, the operative note, the discharge summary, and the surgical pathology report. Baseline variables collected included demographics, comorbidities, pulmonary function, induction therapy use, and the use of $\beta$-blockers. Primary outcomes assessed were postoperative complications, length of stay, and death.

In general, patients considered appropriate for the thoracoscopic approach included those with tumors smaller than $6 \mathrm{~cm}$ in diameter and without evidence of chest wall or central airway involvement on preoperative imaging. However, some patients who would have been candidates for thoracoscopic resection by one surgeon might not have been considered such by all surgeons, and the decision to use either the minimally invasive or open approach was made by the individual surgeon. All patients in this series had an anatomic lobectomy. Excluded were patients who underwent a lesser resection (exploration, wedge, or segmentectomy) or a more extensive operation (pneumonectomy, sleeve lobectomy, chest wall resection, bronchoplasty, or major vascular resection).

Postoperatively, patients were followed by the operative team, including data managers who prospectively entered all complications in the database. All patients underwent continuous electrocardiographic telemetry until discharge from the hospital. Atrial fibrillation (AF) was defined by an irregular rhythm with absent $\mathrm{P}$ waves. Patients were considered to have AF if the episode lasted more than 5 minutes, as determined by means of continuous telemetry. Most cases of AF were confirmed by using 12-lead electrocardiographic analysis. Atelectasis was considered a complication if it prompted bronchoscopy. A prolonged air leak was defined as an air leak present on postoperative day 5. Bleeding was considered a complication if it required reoperation. Pneumonia was defined as meeting 3 of 5 characteristics: fever, leukocytosis, chest radiographic scan with infiltrate, positive culture from sputum, or treatment with antibiotics. The diagnosis of sepsis was recorded for patients with positive blood culture results. Renal failure was defined as an increase of serum creatinine to greater than 2.0 and 2 times the most recent preoperative creatinine level or a new requirement for dialysis postoperatively. Myocardial infarction (MI) was defined by an increase in troponin level to greater than the 99th percentile of the upper reference limit together with at least 1 of the following: symptoms of ischemia, new ST-T changes or new left bundle branch block, development of $\mathrm{Q}$ waves, or imaging evidence of new loss of viable myocardium or new regional wall motion abnormality. The presence of deep venous thrombosis was diagnosed with a lower extremity Doppler study, and the presence of pulmonary embolism was diagnosed with a V/Q scan, angiogram, or spiral computed tomographic scan.

\section{Surgical Technique and Postoperative Care}

In this series all patients underwent complete anatomic lobectomy and mediastinal lymph node dissection. Conventional lobectomy was performed by using a posterolateral thoracotomy without routine sectioning of a rib; the serratus anterior muscle was spared in all patients, and the latissimus dorsi muscle was spared also in a minority of patients. Mediastinal lymph node dissection at thoracotomy and thoracoscopy included the dissection of all hilar lymph nodes and at least 3 ipsilateral mediastinal lymph node stations.

Thoracoscopic lobectomy was performed as previously described. ${ }^{15}$ Briefly, 2 incisions were used in most patients in this series. The thoracoscope was placed in the seventh or eighth intercostal space in the midaxillary line, and an anterior utility incision was used in the fifth intercostal space anteriorly $(4-5 \mathrm{~cm})$. This provided access for complete hilar and mediastinal dissection. Rib spreading, rib cutting, and retractor use were avoided in all patients undergoing thoracoscopic lobectomy. The established criterion for conversion to thoracotomy was an intraoperative finding or the occurrence of an intraoperative event that the surgeon deemed would be managed more effectively with a thoracotomy. Patients in the thoracoscopy group who were converted to thoracotomy intraoperatively were kept in the thoracoscopy group for the purposes of this analysis.

In general, patients were managed postoperatively according to the same care map plan, irrespective of whether the procedures were performed open or thoracoscopically. Management of chest tubes was similar in both groups, with removal if no air leak was present and drainage was less than $250 \mathrm{~mL} / \mathrm{d}$, although there were minor surgeon-specific preferences. Discharge criteria were similar as well.

\section{Statistical Analysis}

Summary statistics were constructed with the use of frequencies and proportions for categorical data and means, medians, and interquartile ranges for continuous variables. We compared the characteristics of patients who underwent their operations through a thoracoscopy with those who had a lobectomy through a thoracotomy. Fisher's tests and $t$ tests were used to assess the relationship between treatment through a thoracoscopy or thoracotomy when data were dichotomous or distributed normally. The Wilcoxon signed-rank test was used for nonnormally distributed data (chest tube days and length of stay).

Patient matching based on propensity scores was used to account for baseline differences between groups. A nonparsimonious logistic regression model, in which lobectomy through a thoracoscopy or thoracotomy was the dependent variable and variables in Table 1 were the independent variables, was constructed. Patients who required conversion to thoracotomy were analyzed by using the intent-to-treat method. With this model, a propensity score quantifying the likelihood of thoracoscopic lobectomy was calculated for all patients. Patients who received a lobectomy through a thoracoscopy

TABLE 1. Patient characteristics in 1079 patients undergoing lobectomy

\begin{tabular}{lccl}
\hline \multicolumn{1}{c}{ Characteristics } & $\begin{array}{c}\text { THOR } \\
(\mathbf{n = 3 8 2})\end{array}$ & $\begin{array}{c}\text { VATS } \\
(\mathbf{n}=\mathbf{6 9 7})\end{array}$ & $\boldsymbol{P}$ value \\
\hline Age, y & $64 \pm 11$ & $67 \pm 10$ & .0005 \\
Male/female sex, n & $215 / 167$ & $344 / 353$ & .03 \\
Preoperative chemotherapy, n (\%) & $102(27)$ & $62(9)$ & .0001 \\
Preoperative radiotherapy, n (\%) & $85(22)$ & $53(8)$ & .0001 \\
HTN, n (\%) & $193(51)$ & $384(55)$ & .16 \\
MI, n (\%) & $27(7)$ & $57(8)$ & .55 \\
CAD, n (\%) & $74(19)$ & $144(21)$ & .64 \\
CHF, n (\%) & $18(5)$ & $30(4)$ & .76 \\
Atrial fibrillation, n (\%) & $17(4)$ & $45(6)$ & .22 \\
DM, n (\%) & $71(19)$ & $99(14)$ & .07 \\
FEV,$\%$ predicted & $67 \pm 19$ & $73 \pm 29$ & .0001 \\
DLCO, \% predicted & $71 \pm 20$ & $78 \pm 30$ & .0001 \\
Preoperative $\beta$-blocker, n (\%) & $73(19)$ & $128(18)$ & .81 \\
Clinical stage, n (\%) & & & $.003 *$ \\
Benign & $29(8)$ & $42(6)$ & \\
1 & $233(61)$ & $529(76)$ & \\
2 & $37(10)$ & $39(6)$ & \\
3 & $54(14)$ & $39(6)$ & \\
4 & $3(1)$ & $3(0.4)$ & \\
Metastasis & $23(6)$ & $42(6)$ & \\
Small cell lung cancer & $3(1)$ & $3(0.4)$ & \\
\hline Din & &
\end{tabular}

Data are presented as means \pm standard deviations, where shown. THOR, Conventional thoracotomy; VATS, video-assisted thoracoscopic surgery; $H T N$, hypertension; $M I$, myocardial infarction; $C A D$, coronary artery disease; $C H F$, congestive heart failure; $D M$, diabetes mellitus; $F E V_{l}$, forced expiratory volume in 1 second; $D L C O$, carbon monoxide diffusion in the lung. ${ }^{*}$ Mantel-Haenszel $\chi^{2}$ test. 
TABLE 2. Anatomic distribution of lobectomies $(n=1079)$

\begin{tabular}{lcc}
\hline \multicolumn{1}{c}{ Anatomic distribution } & $\begin{array}{c}\text { THOR } \\
(\mathbf{n}=\mathbf{3 8 2})\end{array}$ & $\begin{array}{c}\text { VATS } \\
(\mathbf{n}=\mathbf{6 9 7})\end{array}$ \\
\hline Right upper lobectomy, n (\%) & $125(33)$ & $241(35)$ \\
Right middle lobectomy, n (\%) & $27(7)$ & $65(9)$ \\
Right lower lobectomy, n (\%) & $43(11)$ & $108(15)$ \\
Right bilobectomy, n (\%) & $31(8)$ & $12(2)$ \\
Left upper lobectomy, n (\%) & $109(29)$ & $186(27)$ \\
Left lower lobectomy, n (\%) & $47(12)$ & $85(12)$ \\
\hline
\end{tabular}

THOR, Conventional thoracotomy; VATS, video-assisted thoracoscopic surgery.

or thoracotomy were then matched on the basis of their propensity score. By using a greedy 5-to-1 digit-matching algorithm, we matched each patient who received lobectomy through a thoracoscopy with one patient who had his or her operation through a thoracotomy starting with all 5-digit propensity-score matches (ie, to the nearest 0.00001 ) before moving to those with 4 or fewer matches in an iterative process. We gave up if there was no match to at least 1 decimal point.

The matched cohort was evaluated for differences between treatment groups in each of the potential confounding factors. Postoperative outcomes from the matched cohorts were then compared by using the Fisher's test for categorical outcomes and paired $t$ tests or Wilcoxon signed-rank tests for continuous variables. Statistics were performed with SAS 9.1 software (SAS Institute, Inc, Cary, NC). The greedy propensity matching was performed with an SAS macro written by Lori Parsons (accessed in April 2008). ${ }^{16}$

\section{RESULTS \\ Patient Characteristics}

A review of the prospective database between February 1999 and October 2008 identified 1079 patients who were eligible for the study. Of these patients, lobectomy was performed by means of thoracoscopy in 697 and by means of conventional thoracotomy in 382 , and characteristics of the entire group are detailed in Table 1 . There were $32(4.6 \%)$ conversions caused by dense adhesions (14 patients), bleeding (13 patients), technical difficulties (4 patients), and bronchial injury (1 patient); these patients are analyzed within the thoracoscopic lobectomy group.

The mean age was lower in the thoracotomy group (64 vs 67 years, $P=.0005)$. In addition, the thoracoscopic group had significantly more female patients. The thoracoscopic group had better forced expiratory volume in 1 second and carbon monoxide diffusing capacity values. Significantly more patients in the thoracotomy group received preoperative chemotherapy and radiotherapy. The anatomic distribution of lobectomy is represented in Table 2.

\section{Patient Outcome: Unmatched}

Postoperative outcomes for the entire cohort are detailed in Table 3. There were $22(5.8 \%)$ deaths among the 382 patients in the thoracotomy group and $14(2.0 \%)$ deaths among the 697 patients in the thoracoscopic group $(P=.003)$. The causes of death in the thoracotomy group were the following: sepsis ( 7 patients), pneumonia (2 patients), persistent respiratory failure (4 patients), cardiac arrest (3 patients), ischemic bowel and abdominal sepsis (2 patients), cerebrovascular
TABLE 3. Overall postoperative complications $(n=1079)$

\begin{tabular}{|c|c|c|c|}
\hline Complication & $\begin{array}{c}\text { THOR } \\
(\mathbf{n}=\mathbf{3 8 2})\end{array}$ & $\begin{array}{c}\text { VATS } \\
(\mathbf{n}=697)\end{array}$ & $P$ value \\
\hline Atrial fibrillation, $\mathrm{n}(\%)$ & $85(22)$ & $111(16)$ & .01 \\
\hline Atelectasis, n (\%) & $46(12)$ & $34(5)$ & .0001 \\
\hline Prolonged air leak, n (\%) & $73(19)$ & $77(11)$ & .0004 \\
\hline Bleeding, n ( $\%)$ & $5(1.3)$ & $6(1)$ & .53 \\
\hline Transfusion, n (\%) & $47(12)$ & $27(4)$ & .0001 \\
\hline Wound infection, n (\%) & $3(0.8)$ & $1(0.1)$ & .13 \\
\hline Pneumonia, n (\%) & $35(9)$ & $29(4)$ & .001 \\
\hline Empyema, n (\%) & $6(1.6)$ & $4(0.6)$ & .18 \\
\hline Bronchopleural fistula, n (\%) & $4(1)$ & $1(0.1)$ & .06 \\
\hline Sepsis, n (\%) & $10(3)$ & $4(0.6)$ & .008 \\
\hline Renal failure, n (\%) & $19(5)$ & $12(2)$ & .003 \\
\hline CVA, n $(\%)$ & $3(0.8)$ & $3(0.4)$ & .67 \\
\hline MI, n (\%) & $2(0.5)$ & $5(0.7)$ & 1.0 \\
\hline Ventricular arrhythmia, n (\%) & $2(0.5)$ & $5(0.7)$ & 1.0 \\
\hline DVT, n $(\%)$ & $2(0.5)$ & $1(0.1)$ & .29 \\
\hline PE, n (\%) & $3(0.8)$ & $3(0.4)$ & .67 \\
\hline $\begin{array}{l}\text { Chest tube duration, median days } \\
\text { (25th-75th quartile) }\end{array}$ & $4(3-6)$ & $3(2-4)$ & $.0001^{*}$ \\
\hline $\begin{array}{l}\text { Length of hospital stay, median days } \\
\qquad \text { (25th-75th quartile) }\end{array}$ & $5(4-7)$ & $4(3-5)$ & $.0001^{*}$ \\
\hline Death, n $(\%)$ & $22(6)$ & $14(2)$ & .003 \\
\hline Patients with no complication, n (\%) & $192(50)$ & $485(70)$ & .0001 \\
\hline
\end{tabular}

THOR, Conventional thoracotomy; VATS, video-assisted thoracoscopic surgery; $C V A$, cerebrovascular accident; $M I$, myocardial infarction; $D V T$, deep venous thrombosis; $P E$, pulmonary embolism. *Wilcoxon signed-rank test.

event (2 patients), and MI (1 patient). In the thoracoscopic group causes of death were the following: MI (4 patients), sepsis ( 3 patients), cerebrovascular event ( 3 patients), persistent respiratory failure ( 2 patients), and complications of cerebral edema (1 patient).

In the analysis of individual complications in the entire cohort, thoracoscopic lobectomy was associated with a lower incidence of AF $(16 \%$ vs $22 \%, P=.01)$, atelectasis $(5 \%$ vs $12 \%, P=.0001)$, prolonged air leak $(11 \%$ vs $19 \%, P=$ $.0004)$, transfusion ( $4 \%$ vs $12 \%, P=.0001)$, pneumonia (4\% vs $9 \%, P=.001)$, sepsis $(0.6 \%$ vs $3 \%, P=.008)$, renal failure $(2 \%$ vs $5 \%, P=.003)$, and death $(2 \%$ vs $6 \%, P=.003)$. The proportion of patients not experiencing any postoperative complication was higher in the thoracoscopic group $(70 \%$ vs $50 \%, P=.0001)$. A thoracoscopic approach also resulted in few chest tube days (median of 3 vs 4 days, $P<.0001$ ) and shorter length of stay (median of 4 vs 5 days, $P<.0001)$.

\section{Patient Outcomes: Propensity Matching}

A greedy matching algorithm was used to obtain 2 groups with similar baseline characteristics to accurately compare the outcomes of thoracoscopic lobectomy with those of lobectomy by means of thoracotomy. By using this propensity-matched approach, 284 patients from the thoracotomy group were matched and compared with 284 patients from the thoracoscopic group based on propensity scores. The 
TABLE 4. Patient characteristics after propensity score-based matching $(\mathbf{n}=\mathbf{5 6 8})$

\begin{tabular}{lccc}
\hline \multicolumn{1}{c}{ Characteristics } & $\begin{array}{c}\text { THOR } \\
(\mathbf{n}=\mathbf{2 8 4})\end{array}$ & $\begin{array}{c}\text { VATS } \\
(\mathbf{n}=\mathbf{2 8 4})\end{array}$ & $\boldsymbol{P}$ value \\
\hline Age, y & $65 \pm 11$ & $65 \pm 10$ & .86 \\
Male/female sex, n & $159 / 125$ & $155 / 129$ & .80 \\
Preoperative chemotherapy, n (\%) & $50(18)$ & $49(17)$ & 1.0 \\
Preoperative radiotherapy, n (\%) & $35(12)$ & $40(18)$ & .62 \\
HTN, n (\%) & $150(53)$ & $142(50)$ & .56 \\
MI, n (\%) & $20(7)$ & $22(8)$ & .87 \\
CAD, n (\%) & $59(21)$ & $57(20)$ & .92 \\
CHF, n (\%) & $12(4)$ & $12(4)$ & \\
Atrial fibrillation, n (\%) & $13(5)$ & $10(5)$ & .67 \\
DM, n (\%) & $53(19)$ & $47(17)$ & .58 \\
FEV, \% predicted & $68 \pm 19$ & $67 \pm 22$ & .72 \\
DLCO, \% predicted & $72 \pm 20$ & $72 \pm 21$ & .87 \\
Preoperative $\beta$-blocker, n (\%) & $56(20$ & $50(18)$ & .59 \\
Clinical stage, n $(\%)$ & & & $.86 *$ \\
Benign & $22(8)$ & $15(5)$ & \\
1 & $183(64)$ & $199(70)$ & \\
2 & $29(10)$ & $21(7)$ & \\
3 & $31(11)$ & $24(8)$ & \\
4 & $2(1)$ & $3(1)$ & \\
Metastasis & $14(5)$ & $22(8)$ & \\
Small cell lung cancer & $3(1)$ & 0 & \\
\hline
\end{tabular}

Data are presented as means \pm standard deviations, where shown. THOR, Conventional thoracotomy; VATS, video-assisted thoracoscopic surgery; $H T N$, hypertension; $M I$, myocardial infarction; $C A D$, coronary artery disease; $C H F$, congestive heart failure; $D M$, diabetes mellitus; $F E V_{l}$, forced expiratory volume in 1 second; $D L C O$, carbon monoxide diffusion in the lung. *Mantel-Haenszel $\chi^{2}$ test.

model was effective at matching patients because the 2 groups are now nearly identical with respect to measured baseline characteristics (Table 4). The c-statistic for logistic regression used to generate the propensity model was 0.70 .

Postoperative outcomes in these propensity-matched groups are shown in Table 5. There was no significant difference in 30-day mortality in the matched groups. The overall complication rate was lower in patients who underwent thoracoscopic lobectomy: 196 (69\%) of 284 patients who underwent thoracoscopic lobectomy had no complications in comparison with only $144(51 \%)$ of 284 patients who underwent lobectomy by means of thoracotomy $(P=.0001)$. In addition, thoracoscopic lobectomy was associated with a lower incidence of AF (13\% vs $21 \%, P=.01)$, less atelectasis $(5 \%$ vs $12 \%, P=.006)$, fewer prolonged air leaks $(13 \%$ vs $19 \%, P=.05)$, fewer transfusions ( $4 \%$ vs $13 \%$, $P=.002)$, less pneumonia ( $5 \%$ vs $10 \%, P=.05)$, and less renal failure $(1.4 \%$ vs $5 \%, P=.02)$. Finally, thoracoscopic lobectomy was associated with shorter chest tube duration (median of 3 vs 4 days, $P<.0001$ ) and shorter length of hospital stay (median of 4 vs 5 days, $P<.0001$ ).

\section{DISCUSSION}

Many advantages of thoracoscopic lobectomy are well established, including issues surrounding postoperative pain,
TABLE 5. Postoperative complications after propensity score-based matching $(\mathbf{n}=\mathbf{5 6 8})$

\begin{tabular}{|c|c|c|c|}
\hline Complication & $\begin{array}{c}\text { THOR } \\
(\mathrm{n}=\mathbf{2 8 4}) \\
\end{array}$ & $\begin{array}{c}\text { VATS } \\
(\mathbf{n}=\mathbf{2 8 4}) \\
\end{array}$ & $P$ value \\
\hline Atrial Fibrillation, n (\%) & $61(21)$ & $37(13)$ & 0.01 \\
\hline Atelectasis, n (\%) & $34(12)$ & $15(5)$ & 0.006 \\
\hline Prolonged air leak, n (\%) & $55(19)$ & $37(13)$ & 0.05 \\
\hline Bleeding, $\mathrm{n}(\%)$ & $3(1)$ & $3(1)$ & \\
\hline Transfusion, n (\%) & $36(13)$ & $11(4)$ & 0.002 \\
\hline Wound infection, $\mathrm{n}(\%)$ & $3(1)$ & $1(0.4)$ & 0.62 \\
\hline Pneumonia, n (\%) & $27(10)$ & $14(5)$ & 0.05 \\
\hline Empyema, n (\%) & $2(0.8)$ & $2(0.8)$ & \\
\hline Bronchopleural Fistula, n (\%) & $3(1)$ & $1(0.4)$ & 0.62 \\
\hline Sepsis, n (\%) & $6(2)$ & $1(0.4)$ & 0.12 \\
\hline Renal Failure, n (\%) & $15(5)$ & $4(1.4)$ & 0.02 \\
\hline CVA, n (\%) & $3(1)$ & $2(1)$ & 1.0 \\
\hline MI, n (\%) & $0(0)$ & $1(0.4)$ & 0.50 \\
\hline Ventricular arrhythmia, n (\%) & $2(0.8)$ & $2(0.8)$ & \\
\hline DVT, n (\%) & $2(0.8)$ & $0(0)$ & 0.50 \\
\hline PE, n (\%) & $3(1)$ & $1(0.4)$ & 0.62 \\
\hline $\begin{array}{l}\text { Chest Tube } \\
\text { duration, median } \\
\text { days (25th, 75th quartile) }\end{array}$ & $4(3,6)$ & $3(2,4)$ & $0.0001^{*}$ \\
\hline $\begin{array}{l}\text { Length of hospital } \\
\text { stay, median } \\
\text { days (25th, 75th quartile) }\end{array}$ & $5(4,7)$ & $4(3,6)$ & $0.0001^{*}$ \\
\hline Death, n (\%) & $15(5)$ & $8(3)$ & 0.20 \\
\hline $\begin{array}{l}\text { Patients with } \\
\text { no complication, n (\%) }\end{array}$ & $144(51)$ & $196(69)$ & 0.0001 \\
\hline
\end{tabular}

length of hospital stay, pulmonary function, inflammatory response, and recovery. ${ }^{1-3,5-8}$ In addition, thoracoscopic lobectomy is associated with superior delivery of adjuvant chemotherapy, ${ }^{9,10}$ which has been demonstrated to improve survival in patients with breast cancer ${ }^{17,18}$ and thus might also improve survival in patients with lung cancer (although this has not yet been established). A recent review does demonstrate that oncologic outcomes of thoracoscopic lobectomy are equivalent to those of lobectomy by means of thoracotomy. ${ }^{19}$

It has also been recognized that thoracoscopic lobectomy might also be associated with fewer postoperative complications. Compared with recent published results with lobectomy by means of thoracotomy, ${ }^{20}$ morbidity associated with thoracoscopic lobectomy in published series is comparable or superior. ${ }^{2,3,5,7}$ However, it is difficult to draw meaningful conclusions from comparisons of outcomes among series that include only one or the other approach.

The observation that thoracoscopic lobectomy might have a lower complication profile has recently been supported in studies analyzing outcomes of series including patients undergoing thoracoscopic lobectomy and patients undergoing open lobectomy. In one study 122 patients 
undergoing thoracoscopic lobectomy and 122 patients undergoing thoracotomy were compared. ${ }^{12}$ Overall, the incidence of postoperative complications was lower in the thoracoscopic group $(17.2 \%$ vs $27.9 \%, P=.046)$; however, these patients were matched for age and sex only, and there was no significant difference in the incidence of any of the specific complications reported. In a second study focusing on elderly patients (age $\geq 70$ years), a retrospective, matched case-control study was performed evaluating the perioperative outcomes after lobectomy by means of thoracoscopy and thoracotomy. ${ }^{13}$ After matching based on age, sex, the presence of comorbid conditions, and preoperative clinical stage, there were 82 patients in each group. Thoracoscopic lobectomy resulted in a significantly lower rate of complications compared with thoracotomy ( $28 \%$ vs $45 \%, P=.04)$. However, this series was limited to patients with clinical stage I NSCLC, and the incidence of several specific complications analyzed individually was not significantly different between the 2 groups. Whitson and colleagues ${ }^{21}$ analyzed the outcomes of an unmatched group of 147 patients who underwent lobectomy, including 88 by means of thoracotomy and 59 by means of thoracoscopy. Thoracoscopic lobectomy was associated with a lower incidence of pneumonia but no difference in other complications, including blood loss, AF, or number of ventilator-dependent days.

In the current study the outcomes of patients undergoing either thoracoscopic lobectomy $(n=697)$ or open lobectomy $(\mathrm{n}=382)$ were analyzed by using a prospective outcomes database. The hypothesis of the study was that an analysis of a larger population would demonstrate improved outcomes overall in the thoracoscopic group, including improved outcomes with specific complications, such as AF. In the entire cohort of 1079 patients, thoracoscopic lobectomy was associated with a lower incidence of AF, atelectasis, prolonged air leak, transfusion, pneumonia, sepsis, renal failure, overall complications, and death. Conclusions from this comparison are limited because of a number of differences in baseline patient characteristics.

To improve the analysis, a propensity-matching method was used to create 2 groups with similar baseline characteristics based on 13 preoperative variables, including clinical stage, comparing 284 patients who underwent thoracotomy and 284 patients who underwent thoracoscopic lobectomy. The overall complication rate was lower in patients who underwent thoracoscopic lobectomy: $196(69 \%)$ of 284 patients who underwent thoracoscopic lobectomy had no complications in comparison with only $144(51 \%)$ of 284 patients who underwent lobectomy by means of thoracotomy. In addition, thoracoscopic lobectomy was associated with a lower incidence of $\mathrm{AF}(13 \%$ vs $21 \%)$, less atelectasis ( $5 \%$ vs $12 \%)$, fewer prolonged air leaks $(13 \%$ vs $19 \%)$, fewer transfusions $(4 \%$ vs $13 \%)$, less pneumonia $(5 \%$ vs $10 \%)$, less renal failure (1.4\% vs $5 \%)$, shorter chest tube duration (median of 3 vs 4 days), and shorter length of hospital stay (median of 4 vs 5 days).

This study demonstrates that thoracoscopic lobectomy is associated with fewer overall complications than lobectomy by means of thoracotomy by using propensity-matched populations based on preoperative variables and on stage. Patients who underwent thoracoscopic lobectomy had a better chance of having no complications and a lower incidence of $\mathrm{AF}$, an established risk factor for length of stay, and other complications after lobectomy. ${ }^{22}$ In addition, thoracoscopic lobectomy was associated with a lower incidence of atelectasis, prolonged air leaks, transfusions, pneumonia, and renal failure. Consistent with other studies, 2,3,5-8,12,13 thoracoscopic lobectomy was associated with a shorter chest tube duration and length of hospital stay.

There are several implications based on these conclusions. First, the underlying factors responsible for the advantage of the minimally invasive strategy should be analyzed, which might be used to improve the safety and outcomes of other thoracic procedures. Although it could be assumed that rib spreading accounts for increased postoperative pain and that other outcome differences follow, this has not been demonstrated experimentally. ${ }^{23}$ Furthermore, although postoperative pain might influence some outcome variables, there could be other more important factors that relate to the lower incidence of $\mathrm{AF}$ and the higher fraction of patients with no complications.

In addition, the lower complication profile of thoracoscopic lobectomy might improve the development of risk assessment algorithms and patient selection. ${ }^{24}$ Patients who are currently deemed medically inoperable based on age, pulmonary function, or other factors might, in fact, be acceptable candidates for thoracoscopic lobectomy. ${ }^{5,25,26}$ Finally, despite concerns regarding ultimate outcomes after thoracoscopic lobectomy, it is possible that this approach might be associated with superior overall outcomes, considering the improvement in postoperative morbidity demonstrated by this and other studies, ${ }^{12-14}$ as well as the improvement in the delivery of adjuvant therapy. ${ }^{9,10}$

The conclusions of the current study might be limited by the nature of reviewing a single-institution, nonrandomized series, although the propensity-matching approach allows for analysis of similar groups of patients. This study does not recreate the conditions of a prospective randomized trial; it attempts to analyze the outcomes of 2 operative strategies by using propensity matching to minimize bias in the 2 groups. Because this is a retrospective analysis, it is possible that the capturing of complications might be incomplete; however, the analysis used a database that includes complications that are recorded prospectively. It is also possible that other open techniques using anterior thoracotomy might be associated with better outcomes than a posterolateral thoracotomy, as used in this series; however, when compared 
with thoracoscopic lobectomy in other studies, anterolateral thoracotomy was associated with more pain. ${ }^{9}$

In summary, this study suggests that the probability of having no postoperative complications after lobectomy is better with the thoracoscopic approach when compared with the conventional thoracotomy approach. Thoracoscopic lobectomy is also associated with a lower incidence of postoperative AF and several other major complications. These data suggest that the outcomes of patients with early-stage NSCLC would be improved by using the thoracoscopic approach. The underlying factors responsible for this advantage should be analyzed, which might be used to improve the safety and outcomes of other thoracic procedures.

\section{References}

1. Onaitis MW, D'Amico TA. Diagnosis and staging of lung cancer. In: Patterson GA, Cooper JD, Deslauriers J, Lerut AEMR, et al, editors. Pearson's Thoracic and Esophageal Surgery. Philadelphia: Churchill Livingstone; 2008: pp. 751-64.

2. McKenna RJ, Houck W, Fuller CB. Video-assisted thoracic surgery lobectomy: experience with 1,100 Cases. Ann Thorac Surg. 2006;81:421-6.

3. Onaitis MW, Petersen PR, Balderson SS, et al. Thoracoscopic lobectomy is a safe and versatile procedure: experience with 500 consecutive patients. Ann Surg. 2006;244:420-5.

4. Ettinger DS, Akerly W, Bepler G, et al. National Comprehensive Cancer Network (NCCN). Non-small cell lung cancer clinical practice guidelines in oncology. J Natl Compr Cancer Network. 2008;6:228-69.

5. Demmy TL, Curtis JJ. Minimally invasive lobectomy directed toward frail and high-risk patients: a case control study. Ann Thorac Surg. 1999;68:194-200.

6. Nagahiro I, Andou A, Aoe M, et al. Pulmonary function, postoperative pain, and serum cytokine level after lobectomy: a comparison of VATS and conventional procedure. Ann Thorac Surg. 2001;72:362-5.

7. Swanson SJ, Herndon JE, D'Amico TA, et al. Video-assisted thoracic surgery (VATS) lobectomy - report of CALGB 39802: a prospective, multi-institutional feasibility study. J Clin Oncol. 2007;25:4993-7.

8. Nomori H, Horio H, Naruke T, Suemasu K. What is the advantage of a thoracoscopic lobectomy over a limited anterior thoracotomy procedure for lung cancer surgery? Ann Thorac Surg. 2001;72:879-84.

9. Petersen RP, Pham D, Burfeind WR, et al. Thoracoscopic lobectomy facilitates the delivery of chemotherapy after resection for lung cancer. Ann Thorac Surg. 2007;83:1245-9.

10. Nicastri DG, Wisnivesky JP, Litle VR, et al. Thoracoscopic lobectomy: report on safety, discharge independence, pain, and chemotherapy tolerance. J Thorac Cardiovasc Surg. 2008;135:642-7.

11. Boffa DJ, Allen MS, Grab JD, et al. Data from The Society of Thoracic Surgeons General Thoracic Surgery database: the surgical management of primary lung tumors. J Thorac Cardiovasc Surg. 2008;135:247-54

12. Park BJ, Zhang H, Rusch VW, Amar D. Video-assisted thoracic surgery does not reduce the incidence of postoperative atrial fibrillation after pulmonary lobectomy. J Thorac Cardiovasc Surg. 2007;133:775-9.

13. Cattaneo SM, Park BJ, Wilton AS, et al. Use of video-assisted thoracic surgery for lobectomy in the elderly results in fewer complications. Ann Thorac Surg. 2008;85: 231-6.

14. Muraoka M, Oka T, Akamine S, et al. Video-assisted thoracic surgery lobectomy reduces the morbidity after surgery for stage I non-small cell lung cancer. Jpn J Thorac Cardiovasc Surg. 2006;54:49-55.

15. Burfeind WR, D'Amico TA. Thoracoscopic lobectomy. Operat Tech Thorac Cardiovasc Surg. 2004;9:98-114.

16. Parson LS. Reducingbias in a propensity score matched-pair sample using greedy matching techniques. Available at: http://www2.sas.com/proceedings/sugi26/ p214-26.pdf. Accessed May 29, 2009

17. Bonadonna G, Valagussa P, Moliterni A, et al. Adjuvant cyclophosphamide, methotrexate, and fluorouracil in node-positive breast cancer: the results of 20 years of follow-up. N Engl J Med. 1995;332:901-26.

18. Lohrisch C, Paltiel C, Gelmon K, et al. Impact on survival of time from definitive surgery to initiation of adjuvant chemotherapy for early-stage breast cancer. J Clin Oncol. 2006;24:4888-94.
19. D'Amico TA. Long-term outcomes after thoracoscopic lobectomy. Thorac Surg Clin. 2008;18:259-62.

20. Allen MS, Darling GE, Pechet TV, et al. Morbidity and mortality of major pulmonary resection with early stage lung cancer: initial results of the randomized, prospective ACOSOG Z0030 trial. Ann Thorac Surg. 2006;81:1013-20.

21. Whitson BA, Andrade RS, Boettcher A, et al. Video-assisted thoracoscopic surgery is more favorable than thoracotomy for resection of clinical stage I non-small cell lung cancer. Ann Thorac Surg. 2007;83:1965-70.

22. Polanczyk CA, Goldman L, Marcantonio ER, Orav EJ, Lee TH. Supraventricular arrhythmia in patients having noncardiac surgery: clinical correlates and effect on length of stay. Ann Intern Med. 1998;129:279-85.

23. D'Amico TA. Thoracoscopic lobectomy: evolving and improving. J Thorac Cardiovasc Surg. 2006;132:464-5.

24. Bernard A, Ferrand L, Hagry O, et al. Identification of prognostic factors determining risk groups for lung resection. Ann Thorac Surg. 2000;70:1161-7.

25. Burfeind WR, Tong BC, O'Branski E, et al. Quality of life outcomes are equivalent after lobectomy in the elderly. J Thorac Cardiovasc Surg. 2008;136:597-604.

26. Petersen RP, Pham DK, Toloza EM, et al. Thoracoscopic lobectomy: a safe and effective strategy for patients receiving induction therapy for non-small cell lung cancer. Ann Thorac Surg. 2006;82:214-9.

\section{Discussion}

Dr John Mitchell (Denver, Colo). Dr Fullerton, members, and guests, I would like to congratulate Dr Villamizar and his colleagues on a very nice presentation and a well-written manuscript. In this study you describe a lower incidence of postoperative complications after thoracoscopic lobectomy compared with open lobectomy in patients with lung cancer both in a retrospective analysis of a consecutive series of patients undergoing lobectomy and in a propensitymatched analysis. The third portion that you presented today with the propensity matching based on stage was not in the manuscript I received, and therefore I will not address that per se.

I would guess that in the first overall analysis most of the differences in outcome could be explained by differences in the 2 patient groups, such as the percentage undergoing induction therapy. In the matched analysis there was a greater percentage of patients with no complications, less AF, shorter hospital stays, and a shorter but clinically insignificant chest tube duration.

Your findings frankly mirror my own observations with thoracoscopic lobectomy, but I have a few questions for you.

First, I was wondering if you could tell us what effect the location or the type of lobectomy that you performed had on the complication rates. Were right upper lobes associated with greater complication rates than middle lobes or lower lobes or vice versa?

Dr Villamizar. We did not address that in our review. However, both groups underwent the same percentage of right upper lobectomy. In previous series we have not seen an association between the location of the lobectomy and postoperative complications and, in this particular case, with AF.

Dr Mitchell. My second question has to do with the stage. I was going to ask you how the differences in stage between the 2 matched groups had an effect on the outcomes. At least in the matched series of 153 patients each, a majority of the thoracoscopies, not surprisingly, were done in patients with stage I disease. Could you expound a little bit more on how the stage has an effect on the results that you described today?

Dr Villamizar. When we did the propensity-matched analysis, the idea was to have groups that were comparable in terms of baseline characteristics. We did find in that first propensity-matched analysis that the stage was different, and that is why we proceeded to do another matched analysis, this time based on stage. From that 
last matched analysis, it seems that the stage did not affect the incidence of postoperative complications, and it was more the surgical approach that had a bigger effect.

Dr Mitchell. I had always thought that AF seen in conjunction with lobectomy was due to manipulation of the pulmonary veins or dissection at the hilum. These factors should have been the same whether the procedure was done open or thoracoscopically, and therefore how do you account for less AF in the thoracoscopic group?

Dr Villamizar. That is correct. In our institution the dissection is similar whether we do it thoracoscopically or through a thoracotomy. It is unclear at this point what is causing the AF, but there is some literature that supports the idea that in the open thoracotomy group there is a higher cytokine and inflammatory response overall, as well as increasing white blood cell count. It is possible that the cytokines are related to the presence of AF.

Dr Robert Cerfolio (Birmingham, Ala). Congratulations on an outstanding paper that was very well presented. Can you tell me your criteria for chest tube removal and why the patients undergoing open lobectomies are in the hospital for 7 days and have chest tube in for so long, 7 days? I believe this is what has artificially inflated the bad results in your open group. What do you use to take the chest tube out?

Dr Villamizar. If the patient has drainage of less than $200 \mathrm{~mL}$, even on postoperative day 1 , and the patient does not have an air leak, we will remove the chest tube.

Dr Cerfolio. So you use a very low traditional number. You have not increased that number to 300 or 400 or $450 \mathrm{~mL}$, as we have recently published is safe? Is that the thing that is keeping the chest tube in longer in the open group versus the video-assisted thoracoscopic surgery group?

Dr Villamizar. Those are the criteria that we use for postoperative day 1 or 2 . If the patient does not have a leak and the output is greater than $200 \mathrm{~mL}$ but, let us say, less than $400 \mathrm{~mL}$ and the patient had a thoracoscopic approach, the chest tube would be removed on postoperative day 2 anyway. The reason why there is a difference between the 2 groups in removing the chest tube is because in the thoracotomy group the drainage from the chest tube will be greater than 400 or $500 \mathrm{~mL}$ usually.

Dr Cerfolio. This answer shows why the open group did so poorly in terms of length of stay.

Finally, what would you say to someone like me-and you can go ahead and give me the business if you want, which is always fun-who still prefers open lobectomy but sends my patient home on the third or fourth day, most (ie, 99\%) with their chest tubes out, although some go home with their chest tubes in if they have an air leak. I am very happy with a rib-sparing, nervesparing, muscle-sparing thoracotomy, and in fact, I think it even hurts less than my patients undergoing video-assisted thoraco- scopic surgery. What would you say to me to convince me to start doing more video-assisted thoracoscopic surgery lobectomies for patients with cancer?

Dr Villamizar. We know that you have very good experience with the thoracotomy approach, and your results are very good. However, there is strong literature that supports the idea that the thoracoscopic approach is related to a lower incidence of postoperative complications and, in this study, AF. Even if you remove the chest tube on the third day and send your patients home at the same time that you would send home a patient undergoing a thoracoscopic approach, it is possible that the incidence of AF and other complications would be reduced if you were using the thoracoscopic approach.

Dr Douglas Wood (Seattle, Wash). I just want to follow up on Dr Cerfolio's question. Most of the outcome variables that you looked at were not affected by providers; however, the variable of chest tube removal and the variable of hospital discharge are related to provider decisions. Can you comment on potential provider biases that might exist in perceiving that patients can have chest tubes removed earlier or that they can be discharged earlier if they undergo thoracoscopy and how that might influence those outcomes in ways that are not related to the procedure itself but are related to the bias of the providers?

Dr Villamizar. We have noticed over the years that even the way that our surgeons approach postoperative recovery in the thoracotomy group is different based on the experience that we have acquired with the thoracoscopic group. The surgeons used to remove chest tubes at a later day, but now what we see is that the chest tube is removed at an earlier point, which is reflected in this study by only a 1-day difference in the median between the 2 groups. Again, this difference is mostly due to patients who have a larger volume draining from the chest tube in the thoracotomy group.

Unidentified speaker. I have a quick question. Your study shows that this operation can be done. The question is this: Should it be done? At the American Thoracic Society, a paper was presented that showed there is a statistically significant lower number of lymph nodes sampled with a video approach from a very highvolume very well-respected cancer center. Did you look at the number of nodes harvested with each technique, and did you see any differences in the pathologic result?

Dr Villamizar. We did not address this question in this study, but our experience is that the number of nodes removed with the thoracoscopic approach is the same or is not statistically different from the number of nodes removed with thoracotomy. In previous series other groups have also demonstrated this fact, as well as no differences in overall survival between the 2 surgical techniques. The survival is comparable or even better with the thoracoscopic approach. 